\title{
Stock Market Prediction Using Artificial Neural Networks
}

\author{
Bing Yang ${ }^{1, a}$, Hao Jiankun ${ }^{1, b}$ and Zhang Sichang ${ }^{1, c}$ \\ ${ }^{1}$ School of Mathematics and Statistics, Shandong University at Weihai, Weihai 264209, China \\ abingyang@sdu.edu.cn, bhaojiankun1209@163.com, czhangsch125@163.com
}

Keywords: Learning Algorithm, Gradient Search Technique, Neural Networks

\begin{abstract}
In this study we apply back propagation Neural Network models to predict the daily Shanghai Stock Exchange Composite Index. The learning algorithm and gradient search technique are constructed in the models. We evaluate the prediction models and conclude that the Shanghai Stock Exchange Composite Index is predictable in the short term. Empirical study shows that the Neural Network models is successfully applied to predict the daily highest, lowest, and closing value of the Shanghai Stock Exchange Composite Index, but it can not predict the return rate of the Shanghai Stock Exchange Composite Index in short terms.
\end{abstract}

\section{Introduction}

Within the last two decades, there have been many attempts to predict stock prices and price fluctuations with neural networks. [1-5, 7-9]. Financial market is a highly complicate nonlinear system. It is not only variation that has its own regulation, but also influenced by many other factors such as politics, economic situation and investors' psychology. Neural network enjoys the virtue of self-organization and adaptability and can learn economical knowledge from historical datasets, so the neural network is suitable implement to predict stock prices and price fluctuations.

According to the efficient market hypothesis, in the financial market, stock prices rapidly adjust to new information once it becomes public, making the prediction of stock market's movements impossible [2]. This judgment is correct for traditional studies using multiple linear regression analysis. However, stock prices will become predictable if the dynamic and non-linear relationships in sock markets are shown. This is the motivation for applying neural networks to financial time series analysis.

Until now, application of neural networks to predict the movements of financial markets has attracted many researchers' attention. White [13] proposed neural network modeling and learning techniques to search for and decode nonlinear regularities of asset price movements and predicted IBM daily stock prices, Chenoweth [4] studied the trading system based on the future values of daily S\&P 500 index. Zhang [15] carried out various analyses in four kinds Stock including Lujiazui, Fuhua Industrial, Changchun Hualian, Shanghai Petrochemical using BP network with special input data. Wang [12] introduced the sliding window technique and RBF network into non-linear time series, the result was good. Some of these researches merely used the past values of the stock index as the input of neural networks so as to obtain forecast, while others made use of additional financial factors as inputs.

In this study we apply back propagation Neural Network models to predict the daily Shanghai Stock Exchange Composite Index. The learning algorithm and gradient search technique are constructed in the models. We evaluate the prediction models and conclude that the Shanghai Stock Exchange Composite Index is predictable in the short term. Empirical study shows that the Neural Network models is successfully applied to predict the daily highest, lowest, and closing value of the Shanghai Stock Exchange Composite Index, but it can not predict the return rate of the Shanghai Stock Exchange Composite Index in short terms.

This paper is organized as follows. In the next section, we introduce the model of neural networks. Section 3 gives the data description and analysis. In section 4, we evaluate the prediction models. Section 5 summarizes the conclusions. 


\section{Models of Neural Networks}

In this section we describe general features of applications of the Neural Networks to data analysis. The Neural Network is not a standard tool for statistical analysis, which can be regard as an algorithm for Artificial Intelligence and Machine Learning.

The Neural Network has different styles as connection styles are various. Our study chooses Back Propagation Neural Network which uses error back propagation as weight training, to train and test the sample data.

The BP Neural Network is a kind of one-way transmission of multilayer feed forward neural network, with one or more layers of hidden nodes, besides input and output nodes in its structure. There is no connection between nodes on the same level. We can treat it as a highly nonlinear mapping from input to output. Our model uses learning algorithm, gradient search techniques in the learning process and the error back propagation to modify weights, to achieve the minimum of the output error. The following diagram shows a usual BP neural network model with one hidden layer.

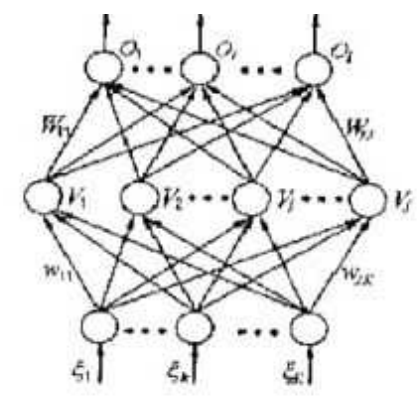

Fig. 1. Structure of BP Neural Networks

Where $O_{i}$ is output unit, $V_{i}$ is the hidden unit, $\xi_{k}$ is input unit, $w_{j k}$ is connection weight from the input unit $k$ to hidden unit $j, W_{i j}$ is connection weight from the hidden unit $j$ to output unit $i$, $\omega=(W, w)$ are all the connections weight. $\mu$ means different input mode, and $p$ is the number input mode, where $\mu=1,2, \cdots, P, g_{1}, g_{2}$ correspond to the appropriate activation functions of hidden and output layer.

For a given input pattern $\mu$, the input to hidden unit $j$ is $h_{j}^{\mu}=\sum_{k=1}^{K} w_{j k} \xi_{k}^{\mu}$, and the output is

$$
V_{j}^{\mu}=g_{1}\left(h_{j}^{\mu}\right)=g_{1}\left(\sum_{k=1}^{K} w_{j k} \xi_{k}^{\mu}\right)
$$

The input to output unit $i$ is

$$
H_{i}^{\mu}=\sum_{j=1}^{J} W_{i j} V_{j}^{\mu}=\sum_{j=1}^{J} W_{i j} g_{1}\left(\sum_{k=1}^{K} w_{j k} \xi_{k}^{\mu}\right)
$$

and the final output is

$$
O_{i}^{\mu}=g_{2}\left(H_{i}^{\mu}\right)=g_{2}\left[\sum_{j=1}^{J} W_{i j} g_{1}\left(\sum_{k=1}^{K} w_{j k} \xi_{k}^{\mu}\right)\right]
$$

Corresponding to any input mode $\mu$ and output unit $i$, the instantaneous error function is defined as

$$
E^{\mu}(\omega)=\frac{1}{2}\left(\xi_{i}^{\mu}-O_{i}^{\mu}\right)^{2}=\frac{1}{2}\left\{\xi_{i}^{\mu}-g_{2}\left[\sum_{j=1}^{J} W_{i j} g_{1}\left(\sum_{k=1}^{K} w_{j k} \xi_{k}^{\mu}\right)\right]\right\}^{2},
$$

and total error function for the output unit $i$ is defined as

$$
E(\omega)=\sum_{\mu=1}^{P} E^{\mu}(\omega)=\frac{1}{2} \sum_{\mu=1}^{P}\left(\xi_{i}^{\mu}-O_{i}^{\mu}\right)^{2} .
$$

The weight learning is to select the appropriate increment as new weight for each $\omega$, so that the conduct of the error function decreases as the iteration, and eventually reach a certain minimum value 
in whole or in part. The gradient descent algorithm can be carried out on the right to learn. For the connection weight matrix $W$ between hidden units and output units and the input mode $\mu$, we can use the rules of the gradient descent to obtain

$$
\Delta W_{i j}=-\eta \frac{\partial E^{\mu}}{\partial W_{i j}}=\eta \delta_{i}^{\mu} V_{j}^{\mu},
$$

where $\eta>0$ is the appropriate selection of learning step, and $\delta_{i}^{\mu}=g_{2}^{\prime}\left(h_{i}^{\mu}\right)\left(\xi_{i}^{\mu}-O_{i}^{\mu}\right)$.

Similarly, for the connection weight matrix between hidden units and input unit $w$, we can use the chain rule to obtain

$$
\Delta w_{j k}=-\eta \frac{\partial E^{\mu}}{\partial w_{j k}}=-\eta \frac{\partial E^{\mu}}{\partial V_{j}^{\mu}} \frac{\partial V_{j}^{\mu}}{\partial w_{j k}}=\eta \delta_{j}^{\mu} \xi_{k}^{\mu},
$$

where $\delta_{j}^{\mu}=g_{1}^{\prime}\left(h_{j}^{\mu}\right) \sum_{i} W_{i j} \delta_{i}^{\mu}$.

Activation functions $g_{1}, g_{2}$ shall be single-valued function, to make neurons reversible. Commonly used functions are the Sigmoid function and the log-Sigmoid function, and the mathematical expressions are

$$
\begin{aligned}
& \text { Sigmoid function: } f(x)=\frac{2}{1+e^{-2 x}}-1=\frac{1-e^{-2 x}}{1+e^{-2 x}}, \\
& \text { Log-Sigmoid function: } f(x)=\frac{1}{1+e^{-x}} .
\end{aligned}
$$

\section{Data Description and Analysis}

Number section and subsection headings consecutively in Arabic numbers and type them in bold. Avoid using too many capital letters. If any further subdivision of a subsection is needed the titles should be 10 point and flushed left.

The data used in this study are obtained from the web site of Shanghai Stock Exchange. Our data covers the horizon from March 17, 2010 to April 28, 2010, including the daily prices of The Shanghai Stock Exchange Composite Index.

The main purpose is to predict the daily highest, lowest, closing, and opening prices of individual stock and the daily return. In order to make the information more comprehensive and more predicable, we choose following indicators as the input variables to neural network: for The Shanghai Stock Exchange Composite Index , the input include the daily opening, highest, lowest, and closing prices, trading volume, difference between the highest and lowest prices, and the daily return; and chose the input include the highest, lowest, opening and closing prices, trading volume, difference between the highest/lowest prices and return for one stock.

The excess return is calculated as: $\sqrt{(\pi / 2)}\left|R_{t}-\mu_{t}\right|$, where $p_{t}$ is the closing price of the $t$ day, and $R_{t}$ is daily return of the Shanghai Stock Exchange Composite index .

The data should be normalized due to the different dimensions. The normalization method for each variable is to divide its maximum value. Thus, we obtain the normalized data within the limit of $[-1,1]$.

Names of each variable after normalization at time $t$ are as following:

For the Shanghai Stock Exchange Composite index : highest price $H_{1 t}$, lowest price $L_{1 t}$, opening price $O_{1 t}$, closing price $C_{1 t}$, trading volumes $V_{1 t}$, difference between the highest and lowest prices $H_{1 t}-L_{1 t}$, and return $R_{1 t}$.

Assuming

$$
x_{t}=\left(O_{1 t}, H_{1 t}, L_{1 t}, C_{1 t}, V_{1 t}, H_{1 t}-L_{1 t}, R_{1 t}\right)^{T}
$$


The input matrix of neural network is $X_{t}$, and the outputs are as follows:

$$
\begin{aligned}
& Y_{1}=\left(H_{t-n+2}, H_{t-n+3}, \cdots, H_{t+1}\right), Y_{2}=\left(L_{t-n+2}, L_{t-n+3}, \cdots, L_{t+1}\right), \\
& Y_{3}=\left(C_{t-n+2}, C_{t-n+3}, \cdots, C_{t+1}\right), \quad Y_{4}=\left(R_{t-n+2}, R_{t-n+3}, \cdots, R_{t+1}\right) .
\end{aligned}
$$

\section{Neural Network Predicting}

Network Settings. The principles of choose the number of hidden nodes are:

(1) $\log _{2}$ (The dimension of input neurons) +1 ;

(2) Generally, select $\sqrt{\left(n_{0}+n_{1}\right)}+n$, where $n_{0}$ is the number of input neurons, $n_{1}$ is the number of output neurons, and $n$ is an integer from 1 to 10 ;

(3) The number of nodes in hidden layer $=($ input nodes + output nodes $) / 2$.

The network has 75 nodes of input units, one node of output units, and then the number of hidden nodes is 38 . We take $10^{-3}$ as the training error, and 1000 as the training steps, choose the log Sigmoid function as hidden layer activation function, and the linear function as output function.

Network Training. Take $n=30$, and train the network respectively. We get the error curves:
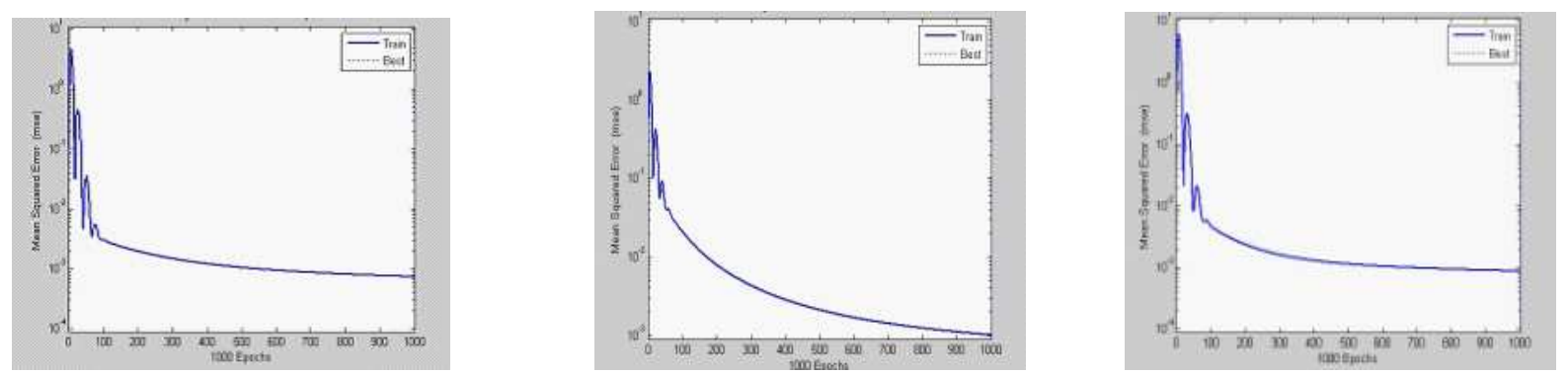

Fig. 2. Error curves of Highest price Fig. 3. Error curves of Lowest price Fig. 4. Error curves of Closing price

While training step is almost 200 steps, errors of $H$ and $C$ have reached $10^{-3}$ respectively, and when training step is 1000 steps, error of $L$ has reached $10^{-3}$. That is a good training effect. The relative error curves of these four indicators are shown as follows:

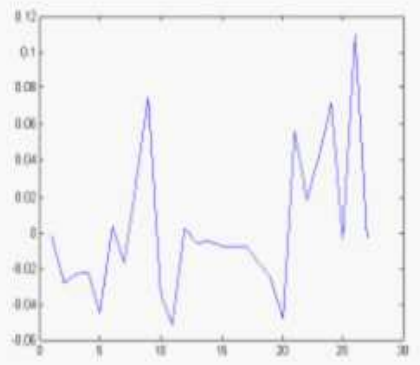

Fig. 5. Relative error of highest price

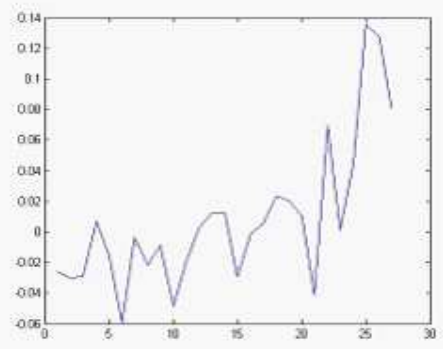

Fig. 7. Relative error of Closing price

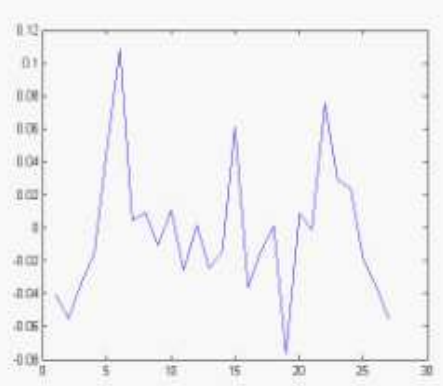

Fig. 6. Relative error of lowest price

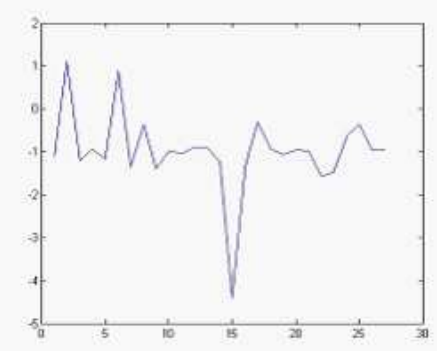

Fig. 8. Relative error of Return 
Access specific data on the training of network test, from Figure 5 to Figure8 we can know that, the relative errors of $H, L$ and $C$ are very small, indicating the accuracy of the network after training is better due to testing, and could be used for prediction. As for $R$, the relative error is large, indicating that this network after training can not be used to predict.

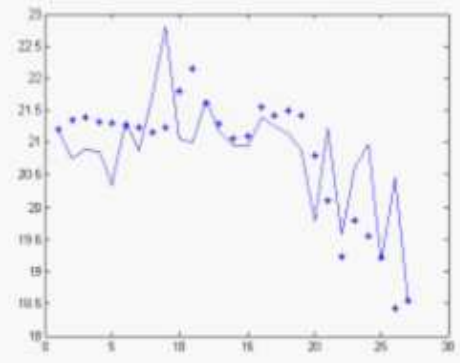

Fig. 9. Fitting effect of highest price

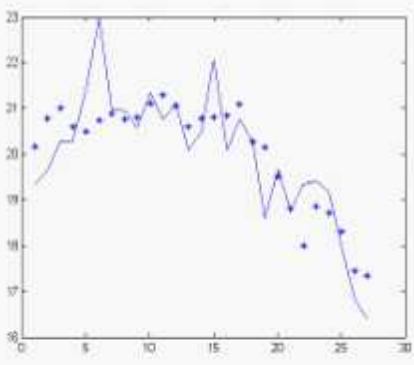

Fig. 10. Fitting effect of lowest price

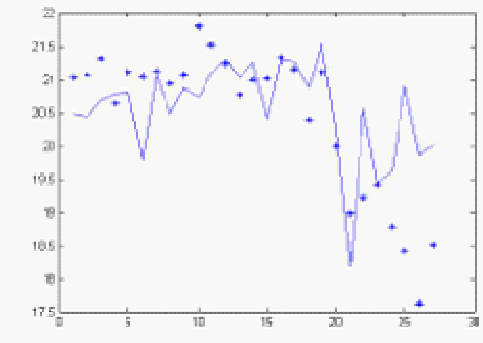

Fig. 11. Fitting effect of Closing price

As Figure9, Figure 10, and Figure 11 show, the network after training can fit the original data, and results of forecast are good. Thus, we conclude that the trained neural network is very suitable for predicting the highest price $H_{1 t}$, lowest price $L_{1 t}$, opening price $O_{1 t}$ and closing price $C_{1 t}$ of the Shanghai Stock Exchange Composite Index:, but the result for the return $R_{t}$ is bad.

\section{Conclusions}

From the present analysis, we can conclude that:

(1) The Neural Network model is an appropriate tool to predict the Shanghai Stock Exchange Composite Index, which means that the Chinese Securities Market is not an efficient market.

(2) Based on the history data, the Neural Network model is successfully applied to predict the daily highest/lowest price and Closing price of the Shanghai Stock Exchange Composite Index in short time, but it is ineffective for predicting the return rate of the Shanghai Stock Exchange Composite Index.

(3) The Neural Network model may help in the further research on derivative products pricing, portfolio management and financial risk management.

\section{References}

[1] H. Ahmadi, Testability of the arbitrage pricing theory by neural networks. In: Proceedings of the international joint conference on neural networks (IJCNN), Washington, DC, June 1990: 385-393.

[2] E. Azoff, Neural network time series forecasting of financial markets, Wiley, New York, 1994.

[3] A. Chen, M. Leungb, H. Daoukc, Application of neural networks to an emerging financial market: forecasting and trading the Taiwan Stock Index, Computers \& Operations Research 30: 901-923, 2003. 
[4] T. Chenoweth, Z. Obradovic and S. Lee, Technical trading rules as a prior knowledge to a neural networks prediction system for the S\&P 500 index, Northcon/95, Portland: 111-115, 1995.

[5] J. H. Choi, M. K. Lee, and M. W. Rhee, Trading S \& P 500 stock index value futures using a neural network. In: Freedman RS (ed) Proceedings of the 3rd annual international conference on artificial intelligence applications on Wall Street, New York, June, 1995: 63-72.

[6] J. Connor, Time Series and Neural Network Modeling, Ph.D. thesis, University of Washington, Seattle, 1993.

[7] M. R. Hassan, B. Nath, M. Kirley, A fusion model of HMM, ANN and GA for stock market forecasting. Expert Systems with Applications 33: 171-180, 2007.

[8] S. H. Kim, S. H. Chun, Graded forecasting using an array of bipolar predictions: application of probabilistic neural networks to a stock market index, International Journal of Forecasting 14: 323-337, 1998.

[9] M. Leung, A. Chen, and H. Daouk, Application of Neural Networks to an Emerging Financial Market: Forecasting and Trading the Taiwan Stock Index, Computers \& Operations Research, July 10, 2001.

[10]B. Tirozzi, S. Puca, S. Pittalis, and A. Bruschi, Neural Networks and Time Series: Reconstruction and Extreme-Event Analysis, Birkhäuser Boston, 2006: 35-57.

[11]R. R. Trippi, D. DeSieno, Trading equity index value futures with a neural network. J Portfolio Manage 19:27-33, 1992.

[12]Wang Shangfei, Zhou Peiling, Application of RBF Network to Stock Prediction, Theory and Methods Research, 6: 44-46, 1997.

[13]H. White, "Economic prediction using neural networks: The case of IBM daily stock returns," IEEE International Conference on Neural networks, San Diego, 2:451-458, 1988.

[14]Xu Bingzheng, Zhang Bailing and Wei Gang, Neural Network Theory and Applications, The Press of South China University of Technology, 1994.

[15]J. Zhang, J. Yong, Neural Networks to Stock Prediction, Computer Engineer, 23(3): 52-55, 1997. 